\title{
Chlorine passivation of grain boundaries in cadmium telluride solar cells
}

\author{
Michael J. Watts, ${ }_{1}^{1}$ Peter Hatton $\odot,{ }^{2}$ Roger Smith $\odot,{ }^{2}$ Tom Fiducia, ${ }^{3}$ Ali Abbas, ${ }^{3}$ Rachael Greenhalgh, ${ }^{3}$ \\ John M. Walls $\odot,{ }^{3}$ and Pooja Goddard $\odot^{1, *}$ \\ ${ }^{1}$ Department of Chemistry, Loughborough University, Loughborough, Leicestershire LE11 3TU, England, United Kingdom \\ ${ }^{2}$ Department of Mathematical Sciences, Loughborough University, Loughborough, Leicestershire LE11 3TU, England, United Kingdom \\ ${ }^{3}$ Centre for Renewable Energy Systems Technology, Loughborough University, Loughborough, \\ Leicestershire LE11 3TU, England, United Kingdom
}

(Received 27 October 2020; accepted 4 February 2021; published 3 March 2021)

\begin{abstract}
Cadmium telluride is the most commercially important second generation thin-film photovoltaic, with a record solar cell conversion efficiency of $22.1 \%$. However as-deposited cells are $<5 \%$ efficient and require a cell activation treatment with $\mathrm{CdCl}_{2}$ at about $400{ }^{\circ} \mathrm{C}$ to reach commercially viable efficiencies. Such a treatment is a routine process during $\mathrm{CdTe}$ module manufacturing. However, the precise mechanisms at work for this remarkable efficiency enhancement are not well understood. In this paper, atomistic modeling techniques are used to improve the fundamental understanding of the structural and electronic properties of CdTe by modeling the effects of chlorine and other elements with their interaction with extended defects and grain boundaries (GBs). Studies at high spatial resolution with nanoscale secondary ion mass spectrometry, transmission electron microscopy (TEM), and energy dispersive $\mathrm{x}$-ray analysis show that chlorine atoms are concentrated at grain boundaries in CdTe after the $\mathrm{CdCl}_{2}$ treatment. Density functional theory calculations show that both $\mathrm{Cl}_{\mathrm{Te}}$ and $\mathrm{Cl}_{i}$ are stabilized at the grain boundaries compared to bulk CdTe. Similar defect formation energies of these defects suggest both will be present at the grain boundaries. As expected, four single-particle levels are present in the $\Sigma 3$ (112) GB band gap, which explains the low efficiencies prior to treatment. $\mathrm{Cl}_{\mathrm{Te}}$ substitutions passivate one of these levels and partially passivate another two. Remarkably, further addition of $\mathrm{Cl}_{i}$ fully passivates the remaining single-particle levels. This passivation of single-particle levels is most likely to be the primary cause of the efficiency enhancement on chlorine treatment. Further to this, alternative halogens were then trialed as activation treatments. All halogens show similar electronic effects and their defect formation energies follow ionic radii trends.
\end{abstract}

DOI: 10.1103/PhysRevMaterials.5.035403

\section{INTRODUCTION}

Thin-film cadmium telluride (CdTe) solar modules are the most commercially successful second generation photovoltaic technology. Low cost is achieved, in part, by using a rapid sublimation process to deposit the polycrystalline thin-film CdTe absorber [1]. This has been modified recently by introducing selenium to the front of the cell in a CdSeTe alloy [2-4]. In comparison with single-crystal devices, the deposition of the polycrystalline absorber introduces a range of defects, including grain boundaries (GBs), which place a limit on the conversion efficiency [5].

A cadmium chloride $\left(\mathrm{CdCl}_{2}\right)$ heat treatment process is essential for the production of high efficiency CdTe solar cells [6-9]. During the treatment, the CdTe absorber layer is exposed to a $\mathrm{CdCl}_{2}$ vapor with the device stack held at an elevated temperature $\left(\simeq 400{ }^{\circ} \mathrm{C}\right)$. This improves carrier lifetimes in the absorber layer and raises device efficiencies from typically $<5 \%$ into the $10-22 \%$ range [8].

High-resolution characterization shows that, following the treatment, chlorine is present at high concentrations in the GBs of the CdTe absorber [10-12]. Figures 1(a) and 1(b) show

\footnotetext{
${ }^{*}$ Corresponding author.
}

nanoscale secondary ion mass spectrometry (NanoSIMS) images of the chlorine distribution before and after the $\mathrm{CdCl}_{2}$ treatment. In Fig. 1(b) the chlorine distribution appears as bright orange regions along the GBs. Corresponding cathodoluminescence (CL) images are shown in Figs. 1(c) and 1(d).

Before $\mathrm{CdCl}_{2}$ treatment [Fig. 1(c)] the grains are small and there is a dark contrast at the GBs, indicating that these are centers for recombination. After the treatment, the grains are larger and there is a lower contrast at GBs indicating a reduction in GB recombination $[7,8]$.

Previous CL measurements and two-dimensional device models have shown that the treatment causes a reduction in recombination velocity at grain boundaries by a factor of $\simeq 5$ [8]. The transmission electron cross-section image of a CdTe GB alongside energy dispersive $\mathrm{x}$-ray (EDX) elemental maps of the same region also shows segregation of chlorine to the GBs and at the CdS junction as shown in Fig. 2.

During the $\mathrm{CdCl}_{2}$ activation, chlorine interstitials are transported along GBs and form a continuous layer at the junction as shown in Fig. 2. In devices incorporating a CdS buffer, the chlorine decorates the small $\mathrm{CdS}$ grain boundaries and reaches the interface with the transparent conductor layer. This diffusion process through the device is rapid and recrystallization has been observed to initiate at the junction in highly columnar sputtered CdTe absorbers [13]. The chlorine in the GBs 

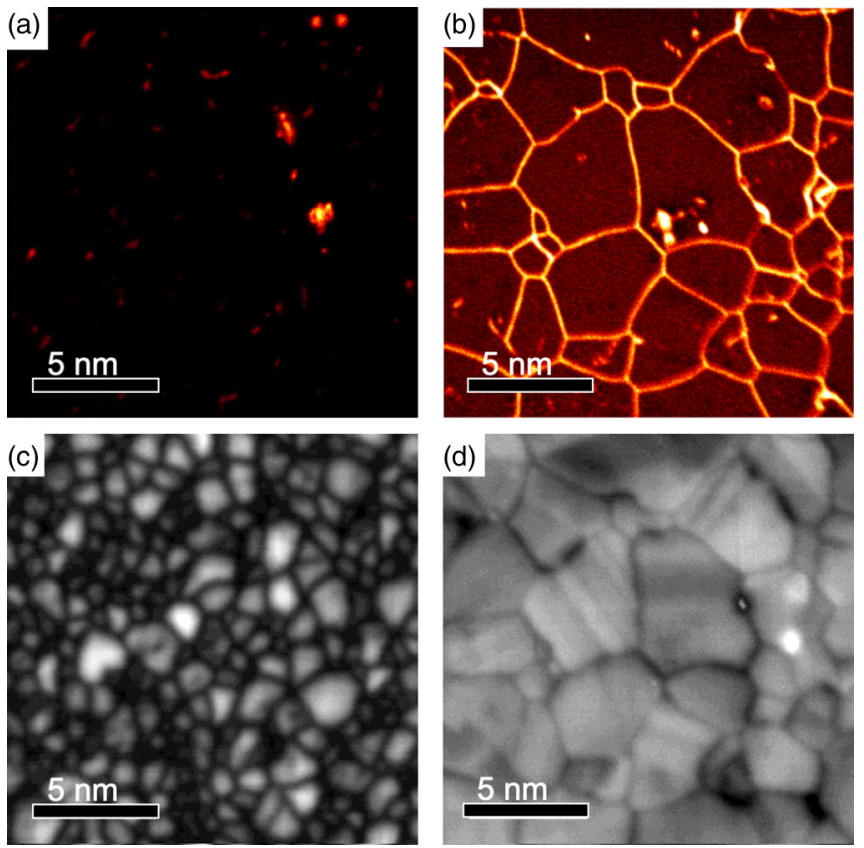

FIG. 1. (a, b) NanoSIMS image of the ${ }^{35} \mathrm{Cl}^{-}$signal distribution on the back surface of the as-deposited CdTe absorber, (a) showing very low signal and some bright spots due to surface contamination and (b) showing chlorine segregation at grain boundaries after $\mathrm{CdCl}_{2}$ activation. (c, d) Map of the cathodoluminescence (CL) intensity on the as-deposited back surface. (c) Lower signal at GBs, i.e., dark GB contrast, indicating high levels of nonradiative recombination relative to grain interiors. (d) Map showing lower GB contrast after $\mathrm{CdCl}_{2}$ activation which indicates reduced GB recombination and hence GB passivation as a result of the segregated chlorine.

passivates defects and there is a direct correlation between the presence of the chlorine in the GBs and the improved electronic properties of the solar cells.

To confirm the electronic effects of chlorine at GBs, density functional theory (DFT) simulations have been carried out. Previous work has shown that GBs introduce defect states into the band gap which would cause recombination. Chlorine substitutions have been shown to passivate some of these energy levels [14-16]. However, many of these calculations
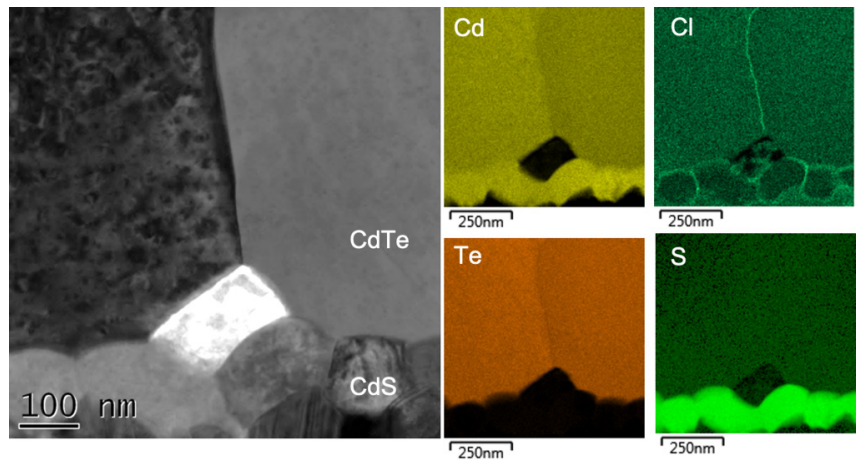

FIG. 2. Cross-sectional TEM image of a CdTe device with corresponding EDX elemental maps for $\mathrm{Cd}, \mathrm{Te}, \mathrm{S}$, and $\mathrm{Cl}$ showing tight segregation of $\mathrm{Cl}$ to the $\mathrm{GB}$ and junction. were performed using DFT functionals which do not accurately predict CdTe's electronic properties due to DFT's well known "band gap problem" [17]. A study of the effect of chlorine on GBs at the hybrid DFT level of theory, which has previously been shown to simulate electronic properties in good agreement with experimental CdTe measurements $[18,19]$, is required to confirm and improve understanding of this passivation mechanism.

Previous work on the $\Sigma 3$ (112) grain boundary by Yang et al. [14] suggests segregation to the boundaries will occur for chlorine interstitials, as interstitial sites in the boundaries are lower in energy than in the bulk. Li et al. [15] investigated the $\Sigma 9 \mathrm{~GB}$ by adding $\mathrm{Cl}_{\mathrm{Te}}$ defects into the structure and showing that segregation will occur. However, diffusion processes through the grain interior (GI) are likely to be dominated by interstitial mechanisms so investigation into the chlorine interstitial segregation is necessary. Furthermore, the direct comparison between the energies of interstitial versus substitution defects has not been shown in any previous work. This is key to understanding the type of defects which will form in these complex structures and is one of the main purposes of this paper.

GBs are very complex and small structural changes can have significant impacts on their properties. The impurity-rich environment of the CdTe solar cell creates many possible defects requiring many individual calculations to investigate. Small model structures must therefore be used, such as the small $\Sigma 3$ and $\Sigma 9$ GBs. It is also important to model both polarities of the GB but several previous studies have focused only on the Te core as this is the most stable [14]. In practice, it is unlikely that all GBs will be Te terminated and the $\mathrm{Cd}$ core has been shown to be more difficult to passivate than the Te core [20]. Adequate calculations have also not been completed to find the defect formation energies of these defect types; doing this will allow for direct comparison of the stable defect types in GIs and GBs.

Most recent studies of electrical properties neglect the possibility of interstitials at GBs. This is due to $\mathrm{Cl}_{i}$ being less stable than $\mathrm{Cl}_{\mathrm{Te}}$ in bulk CdTe [14]. However, in Te-rich conditions the energies of these defects will be shown to be comparable and $\mathrm{Cl}_{i}^{-}$is most stable at higher Fermi energies. This suggests that $\mathrm{Cl}_{i}$ defects at $\mathrm{CdTe}$ GBs require further investigation. If $\mathrm{Cl}_{i}$ is predicted to be present at $\mathrm{GBs}$ this adds another factor to consider when simulating GBs. This paper will seek to perform these calculations to further the understanding of chlorine at CdTe GBs.

It is also suggested from experimental work that other halogens may play a similar role to chlorine in terms of segregation, as seen in Fig. 3, where bromine has clearly segregated to the GBs, and to the device junction. Early experiments also suggest that other halogens may passivate defects at the GB [21]. However, adequate simulations have not been completed to investigate the viability of using other halogens. A preliminary investigation of the effect of other halogens at CdTe GBs is therefore undertaken.

\section{METHODOLOGY}

A $\Sigma 3$ (112) GB was modeled by joining two (111) orientated bulk CdTe grains with one reflected about the $a b$ plane, 


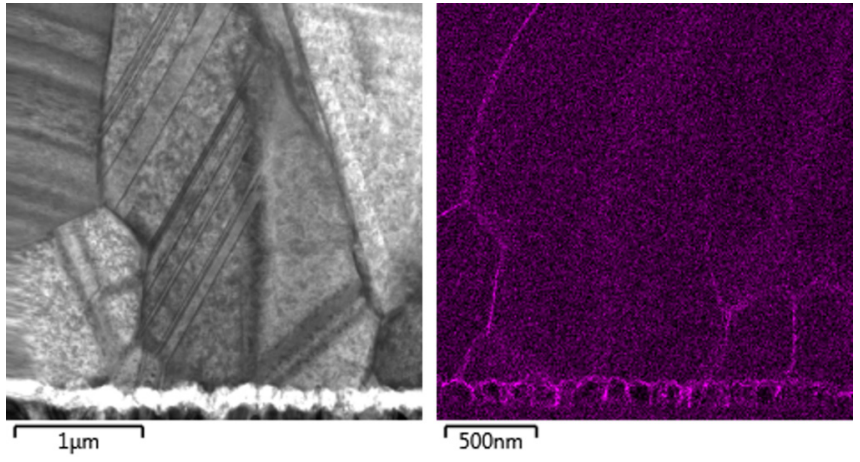

FIG. 3. Cross-sectional TEM image of a CdTe device with a corresponding EDX elemental map for $\mathrm{Br}$ showing segregation of $\mathrm{Br}$ to $\mathrm{GBs}$ and junction.

as shown in Fig. 4. Cd and Te are represented by blue and red atoms, respectively. The cell contains 276 atoms with equal numbers of $\mathrm{Cd}$ and $\mathrm{Te}$ atoms and measures $13.74 \times 62.08 \times$ $11.25 \AA$. There are two different GB structures in the system known as the $\mathrm{Cd}$ core and the Te core. Each is a dislocation core with a "dangling bond" shown by horizontal black lines in Fig. 4. These dislocation cores are separated by $31 \AA$ of zinc-blende CdTe. This length was methodically increased until the defect formation energy (DFE) of chlorine plateaued in the center to give a bulklike environment for the defect. The other dimensions of the cell were also investigated to ensure the trend in halogen DFE replicated that in a $2 \times 2 \times 2$ bulk zinc-blende CdTe supercell containing 64 atoms. The $k$-point grid used to model this GB is $4 \times 1 \times 4$. DFT calculations were carried out using the PBEsol functional, which has been used previously for CdTe systems [19,22].

Although there are three "lines" of CdTe dumbbells in the grain interiors these will become equivalent far from the GBs where the system regains bulklike properties. The central positions of each line were tested and compared with no significant difference found. Therefore only the line of sites highlighted in Fig. 4 was used to represent the grain interior.

Each of the dislocation cores is symmetrical about the dashed vertical lines through their centers in Fig. 4. This means there are three unique sites for each element in each dislocation core. These sites are denoted by a three character reference: the type of core ( $\mathrm{C}$ for $\mathrm{Cd}$ core, $\mathrm{T}$ for $\mathrm{Te}$ core), the element occupying the site in the clean state $(\mathrm{C}$ for $\mathrm{Cd}$, $\mathrm{T}$ for $\mathrm{Te}$ ), and a number 1-3 from bottom to top of the core. All

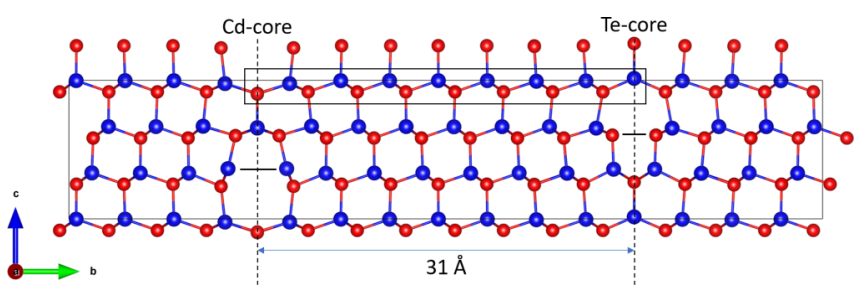

FIG. 4. Structure of the $\Sigma 3$ (112) GB. The GB structures are indicated by vertical dashed lines and the "dangling bonds" are highlighted by horizontal black lines. The line through the grain interior used for segregation studies is shown by the black box. $\mathrm{Cd}$, blue; Te, red. (a)

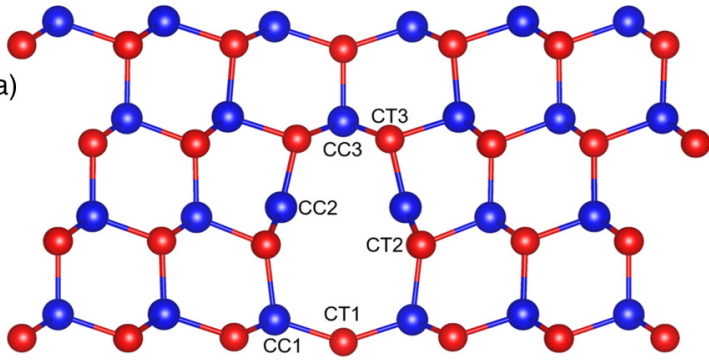

(b)

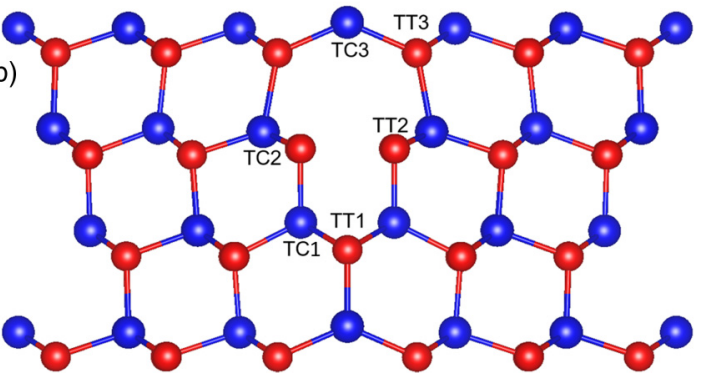

FIG. 5. Labeled Te substitution sites in the (a) Cd core and (b) Te core of the $\Sigma 3$ (112) GB.

sites are labeled in Figs. 5(a) and 5(b) for the Cd and Te cores, respectively.

Each of these sites has a unique coordination. TT1 and $\mathrm{CC} 3$ are tetragonal pyramidal coordinated while TC3 and CT1 are triangular. TT2 and CC2 were equivalent when the structure was made but $\mathrm{CC} 2$ relaxes to triangular coordination while TT2 remains tetrahedral-like with a vacancy. All other sites are distorted tetrahedra. It is likely that different dopant elements will prefer different sites depending on their coordination preference. The TT2 and CC2 sites are undercoordinated and interact across the GB. Tong and McKenna [23] also describe another interaction between CT3 sites which creates single-particle levels within the band gap even though these atoms are correctly coordinated due to their close proximity.

To investigate chlorine segregation to GBs, single chlorine impurity atoms were introduced into the $\Sigma 3$ (112) model GB and relaxed. The position of the defect was varied from the $\mathrm{Cd}$ core to the $\mathrm{Te}$ core through the bulklike separating region shown in Fig. 4. The nearest-neighbor sites to each of the dislocation cores were also included as these sites are often distorted slightly by the GB and therefore do show some differences between them. All possible Te substitutions at both the $\mathrm{Cd}$ and $\mathrm{Te}$ cores were included but $\mathrm{Cd}$ substitutions were not investigated as they are not expected to form.

The DFE calculation is shown in Eq. (1) where $\Delta H^{q}$ is the DFE in a specific charge state, $E_{\text {defect }}$ is the energy of the system containing the defect, $E_{\text {clean }}$ is the energy of the defectfree (clean) system, $n_{i}$ is the number of atoms of element $i$ which have changed between the defect and clean cells, $E_{i}$ is the energy of a single atom of element $i, \mu_{i}$ is the chemical potential of element $i$ in the system, $q$ is charge, $\epsilon_{\mathrm{VBM}}$ is the valence-band maximum (VBM) energy of the clean system, $\Delta E_{F}$ is the Fermi level referenced to $\epsilon_{\mathrm{VBM}}$, and $\Delta_{v}$ is a charge 
correction term:

$$
\begin{aligned}
\Delta H^{q}= & E_{\text {defect }}-E_{\text {clean }}-\sum_{i}\left(n_{i} E_{i}+n_{i} \mu_{i}\right) \\
& +q\left(\epsilon_{\mathrm{VBM}}+\Delta E_{F}+\Delta v\right) .
\end{aligned}
$$

The segregation of chlorine to GBs is only one component of its effects in CdTe. A study of chlorine's effects on GBs at the hybrid DFT level of theory is required to confirm and improve understanding of passivation mechanisms. The large size of the cell used for DFE calculations (276 atoms, $4 \times$ $1 \times 4 k$-point grid) makes HSE06 calculations prohibitively expensive. An equivalent cell with reduced grain width and depth, measuring $9.14 \times 46.30 \times 11.25 \AA$ and containing 136 atoms, is therefore used for electronic properties only. Static calculations with $4 \times 1 \times 2 k$-point grids were used with this smaller cell to reduce further the computational expense.

\section{CHLORINE AT GRAIN BOUNDARIES}

\section{A. Segregation energies}

The segregation curves of chlorine in the $\Sigma 3$ (112) GB in Cd-rich and Te-rich conditions in the neutral charge state are shown in Figs. 6(a) and 6(b), respectively. The $x$ axis refers to the horizontal distance from the center of the Cd-core structure to the defect atom. The $\mathrm{Cd}$-core and Te-core regions are shaded in gray while the unshaded region represents the grain interior.

There are negative DFE values present in the curves. In a bulk system this cannot occur as this would imply the defect is more stable than bulk and would form spontaneously. However, in this case the clean reference cell is the GB shown in Fig. 4 which is already defective. Negative DFE in this case means only that the newly added defect has stabilized the existing GB dislocation core.

Both substitutional and interstitial defects exhibit a strong segregation to both GBs, in agreement with experiment and previous simulation work $[14,23]$. The two defects' segregation curves are first considered separately. $\mathrm{Cl}_{\mathrm{Te}}$ defects have segregation energies of $0.863 \mathrm{eV}$ to the $\mathrm{Cd}$ core and $1.151 \mathrm{eV}$ to the $\mathrm{Te}$ core. These values are in good agreement with previous work $[14,23]$. There is a small decrease in DFE at 25 Å compared to its neighbors. This is at a GB nearest-neighbor site and therefore is subject to some structural distortion from the GB. A similar reasoning explains the larger DFE decrease at $5 \AA$. This is consistent with the tight segregation observed in experiment [10] and is similar to values calculated in previous work [15,24].

Segregation of $\mathrm{Cl}_{i}$ to the $\mathrm{Te}$ core and $\mathrm{Cd}$ core was found to be 2.065 and $1.882 \mathrm{eV}$, respectively. Both of these values are much larger than the corresponding values for $\mathrm{Cl}_{\mathrm{Te}}$. While the DFE plateaus by $4.40 \AA$ from the Cd core, suggesting very tight segregation to this core, defects close to the Te core do not reach a bulklike value until $12.72 \AA$ into the GI.

Due to the DFE method employed, the two curves are now directly comparable. In Cd-rich conditions, interstitials are around $1 \mathrm{eV}$ less stable in the $\mathrm{GI}$ than $\mathrm{Cl}_{\mathrm{Te}}$, which is consistent with bulk results using hybrid functionals [14]. However, the stronger segregation energy of the interstitials means that the DFEs of the two defects are very similar at GBs, with only
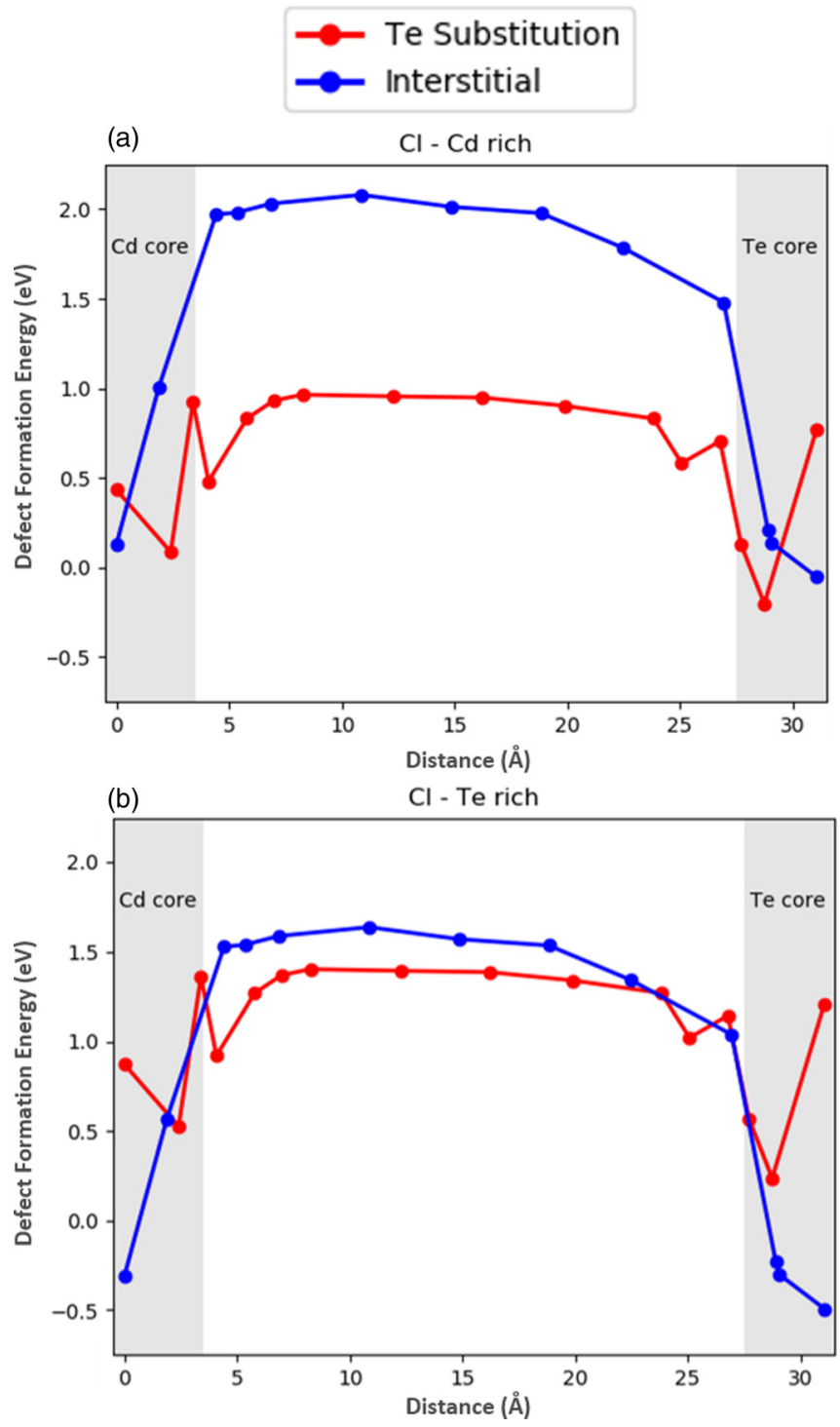

FIG. 6. DFE of single chlorine atoms as Te substitutions and as interstitials in the $\Sigma 3$ (112) GB structure in (a) Cd-rich and (b) Terich conditions.

one substitution site in each GB more stable than the interstitials. This suggests both interstitials and Te substitutions will be present at GBs. This has not been considered previously. When moving from Cd-rich to Te-rich conditions, $\mathrm{Cl}_{\mathrm{Te}}$ defects become less stable while $\mathrm{Cl}_{i}$ becomes more stable. This means interstitials are now significantly lower in energy than substitutions, indicating they will form in preference to substitutions in Te-rich conditions.

\section{B. Electronic properties}

The band structure along the line $\Gamma-L$ in the Brillouin zone and the density of states (DOS) of clean bulk CdTe are shown in Fig. 7 as a reference. The DOS in all figures is normalized to a single atom. The predicted band gap is $1.53 \mathrm{eV}$ at the $\Gamma$ point. The radii of the circles forming each band in the band structure denote their relative contribution from the element defined by its color. It is clear that the valence band 


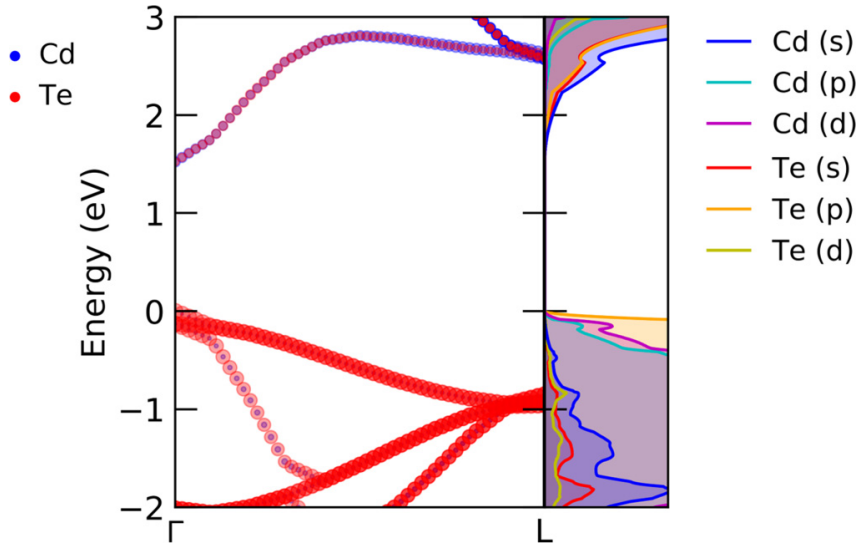

FIG. 7. Band structure ( $\Gamma-\mathrm{L})$ and normalized DOS of clean bulk CdTe.

is primarily formed by Te atoms, which the DOS attributes to Te- $p$ orbitals. The conduction band has similar magnitude contributions from Cd- $s$ and Te- $s$ orbitals.

The corresponding band structure and DOS of the clean GB are shown in Fig. 8. The DOS is also shown in greater detail in Fig. 9(a). Compared to the clean bulk CdTe DOS and band structure shown in Fig. 7, several single-particle energy levels are present in the GB's band gap. Two isolated peaks within the band gap in the DOS are composed largely of Te- $p$ orbitals. The band structure shows that these levels are very flat in the Brillouin zone. There is another energy level which is close to the two flat levels at the $\Gamma$ point but bends into the valence band at $L$. This is the origin of the small flat region in the DOS extending from the valence band. While the shape of this curve is similar to those below it in the valence band, its position at $\Gamma$ suggests this is not part of the valence band. The VBM is therefore one of the several energy levels below this level. Using the highest energy of these levels as the reference VBM, the positions of these energy levels at the $\Gamma$ point are $\mathrm{VBM}+0.239,+0.358$, and $+0.517 \mathrm{eV}$, respectively. All of these energy levels are occupied in both spin channels but only spin-up data are shown for clarity.

There is no unoccupied energy level at $\mathrm{VBM}+1.53 \mathrm{eV}$ to form the bulk band-gap value. When comparing the conduc-

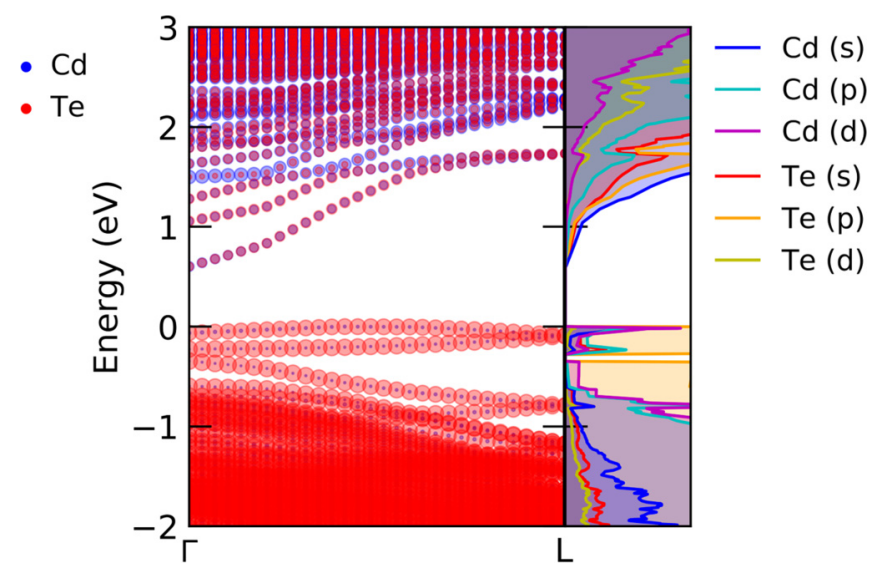

FIG. 8. Band structure ( $\Gamma-\mathrm{L})$ and normalized DOS of the clean GB tion band at $L$ between the bulk and GB plots there are a number of energy levels which have moved to positions lower in energy in the GB, splitting off from the conduction band. One of these levels forms the lowest-energy unoccupied level at the $\Gamma$ point but the lack of connection to the conduction band suggests this is a single-particle defect level. This energy level lies at VBM+1.180 eV and would be damaging to cell performance by increasing recombination. This level is made up of the same orbitals as the bulk conduction band.

The closest energy level to the bulk conduction-band minimum (CBM) values lies at VBM+1.635 eV. It is difficult to determine the position of this level at $L$ due to the overlap of bands in the $\Gamma-L$ high-symmetry line in the band structure. If one assumes this originates from the bulklike conduction band, and is therefore the true CBM, the band gap of the GB is $1.635 \mathrm{eV}$, which is significantly higher than the bulk value and experimental observations [25].

The physical location of these single-particle levels was investigated using partial charge densities. The charge density isosurfaces of the energy levels are shown in Figs. 10(a)10(d). The VBM+0.239-eV level is attributed to the Te-Te interactions between CT3 sites in the Cd core, as denoted in Fig. 5. This is interesting because both of these sites are fully coordinated. Although the neighboring $\mathrm{Cd}$ atom is five coordinated, the level is primarily composed of Te- $p$ orbitals and so does not originate from this $\mathrm{Cd}$ atom.

Both the VBM+0.358- and +0.517-eV levels are attributed to the Te-Te interaction between TT2 sites in the Te core. The second of these levels extends further into the grains than its partner. These sites are undercoordinated so there is expected to be an erroneous interaction at this location which gives rise to these energy levels. The VBM+1.180-eV energy level is spread over several atoms of both elements in the Te core but the largest proportion of this level's isosurface is located around the $\mathrm{Cd}-\mathrm{Cd}$ interaction between TC3 atoms. This site is also undercoordinated.

These results agree qualitatively to those of Tong and McKenna [23] but the positions of these energy levels are different. Tong and McKenna identify two occupied energy levels at $\mathrm{VBM}+0.15$ and $\mathrm{VBM}+0.74 \mathrm{eV}$ which are attributed to the Te-Te interactions in the $\mathrm{Cd}$ and Te cores, respectively. This may be due to the different structural relaxation functionals used.

Tong and McKenna [23] also show that small changes to the distance between defect atoms in their $\Sigma 5$ (310) GB cause the position of the single-particle defect levels to change significantly so this may explain the differences in the positions of the levels found in the $\Sigma 3$ (112) GB between the two works.

All of the identified energy levels within the band gap could be harmful to cell performance by increasing recombination. However, the levels furthest towards the center of the band gap will be most damaging as these are more likely to undergo Shockley-Reed-Hall recombination [26]. Passivating the defects creating these energy levels is therefore an important factor in improving cell performance. As three of the four single-particle levels found in this paper are due to $\mathrm{Te}-\mathrm{Te}$ interactions, doping with competing anions is the logical way to passivate these defects.

The lowest-energy sites for $\mathrm{Cl}_{\mathrm{Te}}$ defects are TT2 and CT3, as defined in Fig. 5. HSE06 calculations of $\mathrm{Cl}_{\mathrm{Te}}$ at these 


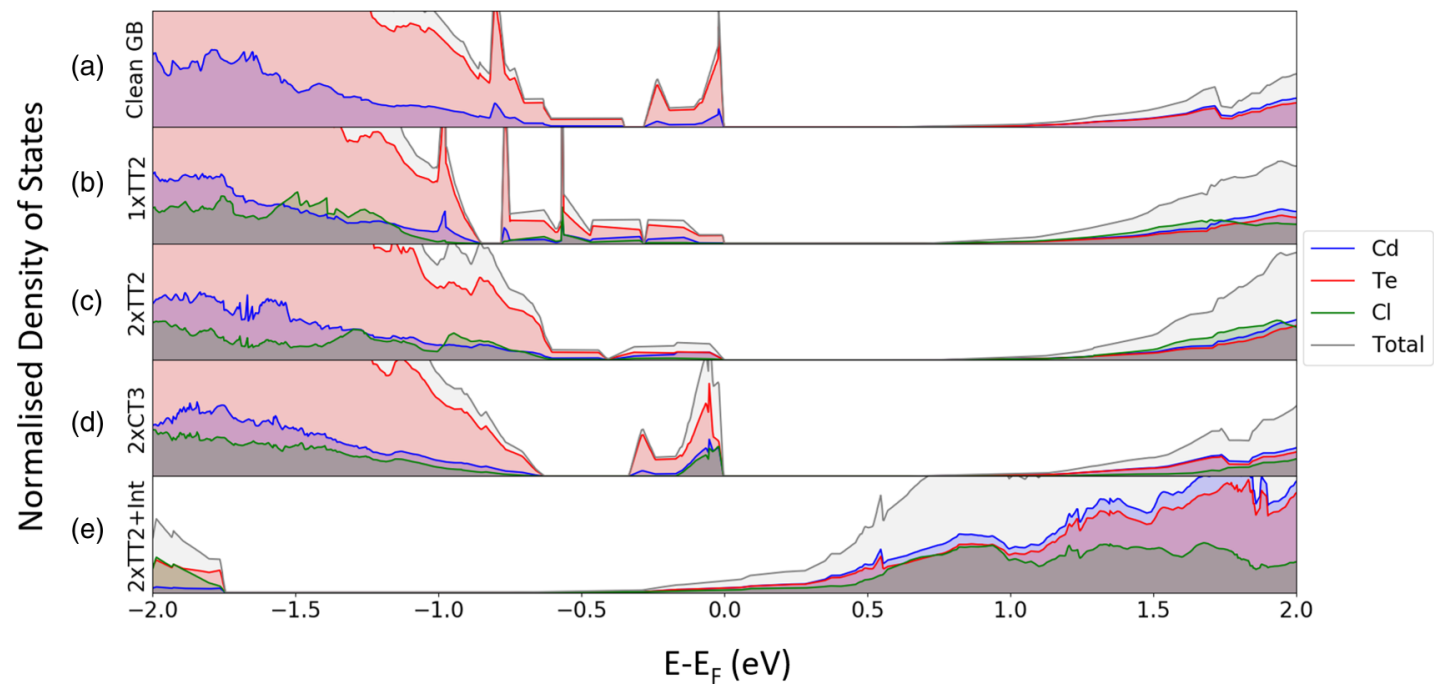

FIG. 9. Normalized DOS of (a) clean $\Sigma 3$ (112) GB with (b) one $\mathrm{Cl}_{\mathrm{Te}}$ defect on the TT2 site, (c) two $\mathrm{Cl}_{\mathrm{Te}}$ defects on the TT2 site, (d) two $\mathrm{Cl}_{\mathrm{Te}}$ defects on the CT3 site, and (e) two $\mathrm{Cl}_{\mathrm{Te}}$ defects on the TT2 site and one $\mathrm{Cl} i$ in the Te core. The Fermi level of each individual structure is used to align the energy scales.

sites were therefore performed. The smaller GB structure used for HSE06 calculations is two atomic layers thick, meaning there are two Te-Te interactions to passivate. If only one of the TT2 sites is substituted for chlorine, the DOS structure becomes more complex, as shown in Fig. 9(b). More singleparticle energy levels are present, which may actually make this structure more damaging than with no chlorine present. This result suggests that there will be a critical amount of chlorine required to passivate a GB.

However, substituting two TT2 sites, effectively creating two $\mathrm{Cl}$-Te bonds, produces a DOS resembling the clean GB DOS, but with a big reduction of the DOS peaks corresponding with the VBM+0.358- and +0.517-eV energy levels. This DOS is shown in Fig. 9(c). The reduction in amplitude of these peaks is evidence of defect passivation. Although there are still some available energy levels at this position, which means some recombination remains, the number of levels available for electrons to occupy is much reduced, which would reduce

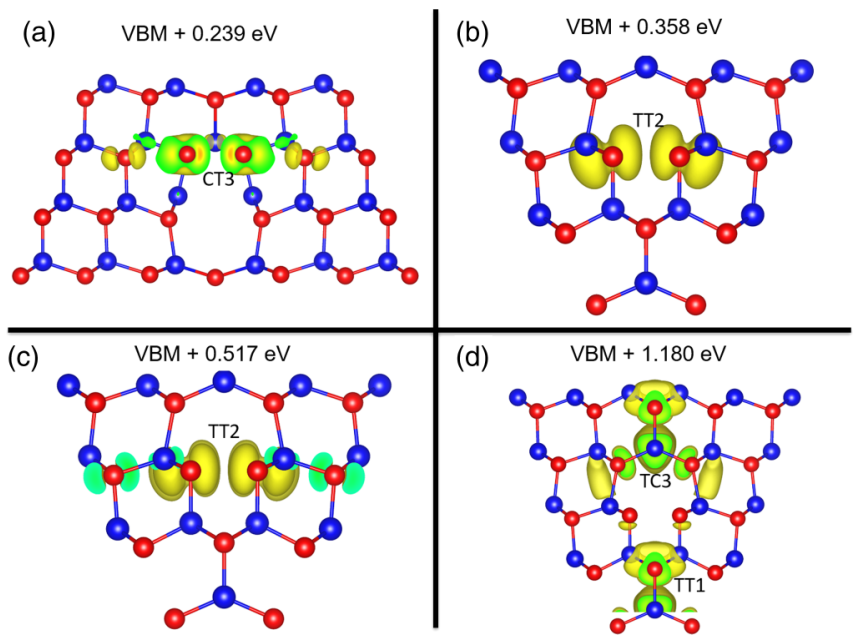

FIG. 10. Partial charge densities of single-particle energy levels identified in the clean $\Sigma 3$ (112) GB. the amount of recombination at this boundary and lead to a higher cell efficiency.

The small area extending from the VBM in the DOS which is due to the VBM+0.239-eV energy level is still present in the calculations containing two $\mathrm{Cl}_{\mathrm{Te}}$ defects on TT2 sites. This is because this level is associated with the CT3 site in the $\mathrm{Cd}$ core and is therefore not affected by substitutions in the Te core. The DOS of two $\mathrm{Cl}_{\mathrm{Te}}$ substitutions at CT3 is shown in Fig. 9(d). The VBM+0.239-eV density has been eliminated, which means this single-particle level has been fully passivated. The VBM +0.358 - and $+0.517-\mathrm{eV}$ levels are unaffected by the $\mathrm{Cl}_{\mathrm{Te}}$ on the CT3 sites so chlorine needs to be present in both dislocation cores to passivate all defects.

So far only $\mathrm{Cl}_{\mathrm{Te}}$ defects have been added. However then only one of the four single-particle levels identified in the $\Sigma 3$ (112) GB has been fully passivated. Two more have been partially passivated but some defect density remains which will continue to cause recombination. This means that some further mechanism is required to explain how the presence of chlorine can reduce the recombination so efficiently as observed experimentally $[7,8]$.

The unoccupied energy level at VBM+1.180 eV is associated with the Cd-Cd interaction at TC3, as seen in Fig. 10. The partial charge density of the levels remaining after TT2 substitution also exhibits significant charge density at this location, as shown in Fig. 11(a). This site is triangular coordinated, meaning there is effectively a Te vacancy at this position. Viewing the partial charge density isosurface from below, as shown in Fig. 11(b), shows that the primary area of electron density is related to this vacancy. The $\mathrm{Cl}_{i}$ is most stable at this position where it occupies the vacancy position. The DOS of a structure containing two $\mathrm{Cl}_{\mathrm{Te}}$ on the TT2 sites and one $\mathrm{Cl}_{i}$ in the Te-core Te-vacancy site is shown in Fig. 9(e), showing that the remaining single-particle levels have been fully removed.

The DOS plots in Fig. 9 are aligned by the calculated Fermi level of each structure. In the 2 xTT2+interstitial case [Fig. 9(e)] the Fermi level has moved into the conduction band and there are some occupied levels above the apparent CBM. 
(a)

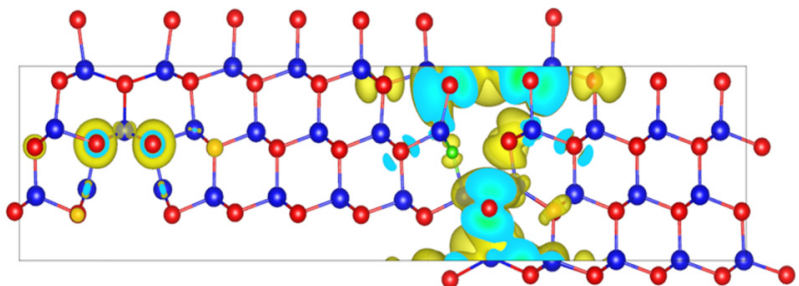

(b)

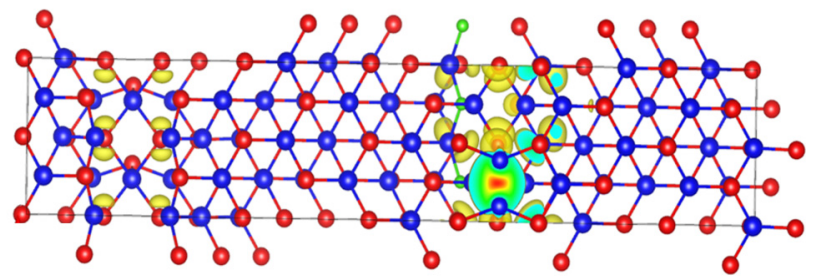

FIG. 11. Partial charge density of the single-particle levels remaining after $2 \times \mathrm{TT} 2 \mathrm{Cl}_{\mathrm{Te}}$ substitution (a) from the conventional side view and (b) from below.

This suggests the addition of the interstitial dopes the GB region $n$ type. This agrees with the DFT results of Li et al. [15].

(a)

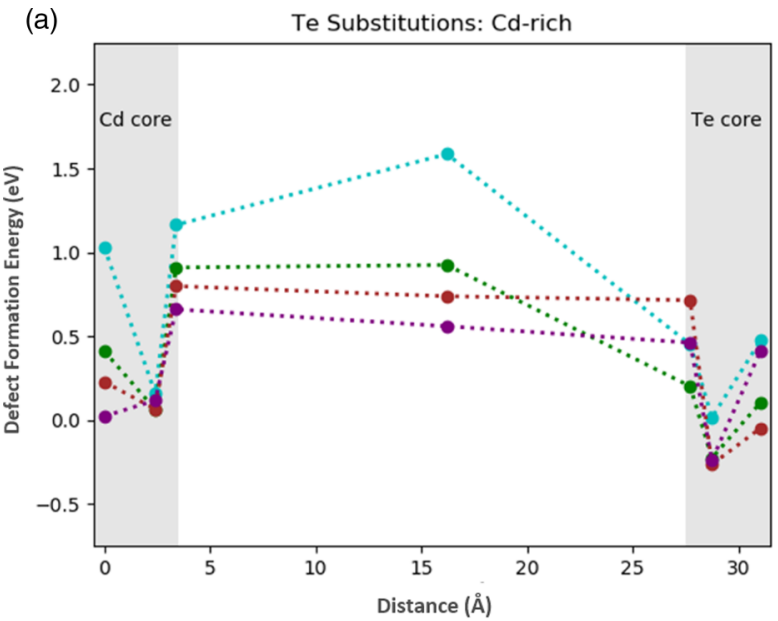

(b)

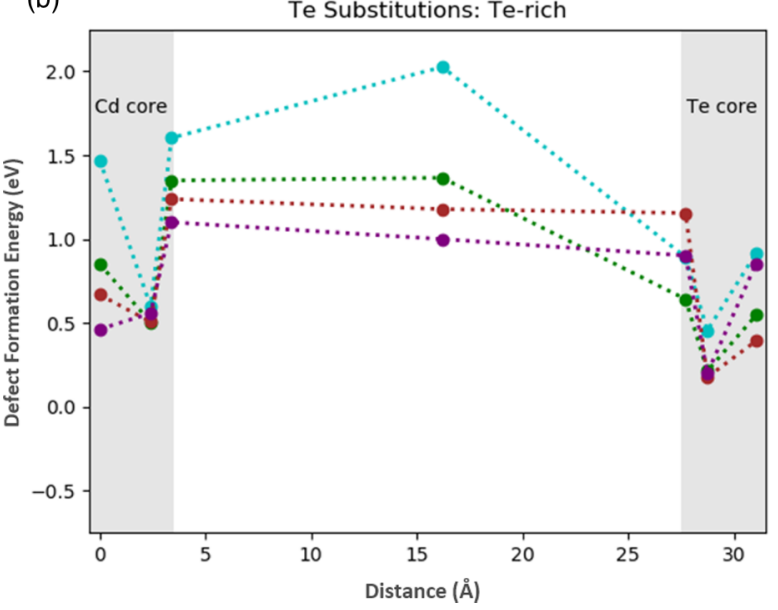

By comparing Fig. 9(e) with the clean GB case in Fig. 9(a) it is clear that chlorine is passivating defects at the GB and that both $\mathrm{Cl}_{\mathrm{Te}}$ and $\mathrm{Cl}_{i}$ are required for complete passivation.

\section{ALTERNATIVE HALOGEN ACTIVATION TREATMENTS}

A simplified version of the chlorine GB segregation study is performed for alternative halogen treatments, $\mathrm{F}, \mathrm{Br}$, and $\mathrm{I}$. All GB sites were calculated but only the centermost position was used to represent bulk CdTe. This means the segregation distance is not determined but can be assumed to be similar to chlorine. The segregation plots for substitutions are shown in Figs. 12(a) and 12(b), interstitials are shown in Figs. 12(c) and $12(\mathrm{~d})$.

All halogens segregate to both GB cores as both interstitials and substitutions. For substitutions, all are most stable at the TT2 site in the Te core and all but iodine are most stable at the CT3 site in the Cd core. Iodine is most stable at the CT1 site, which has triangular coordination and the largest Cd-halogen distance and may favor iodine due to its larger size. The defect formation energies of all halogens at TT2 and CT3 are remarkably similar. In sites CT1, CT2, and TT3 and

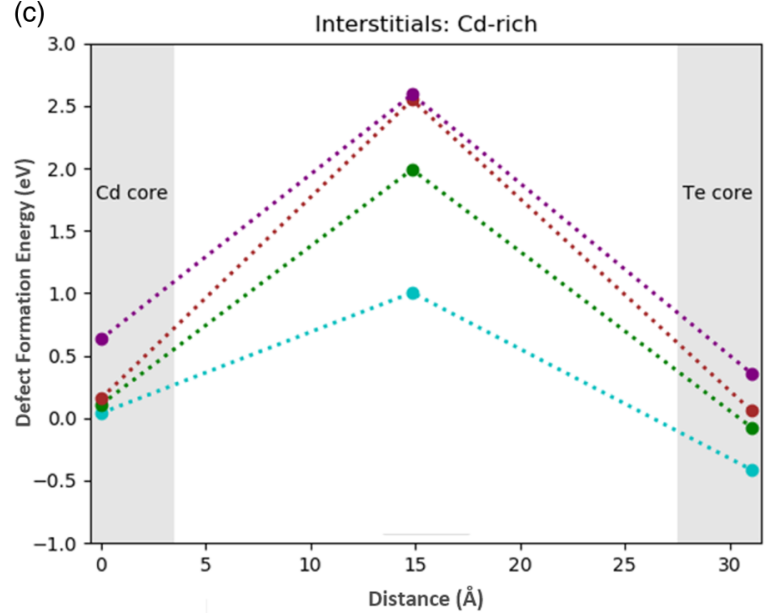

(d)

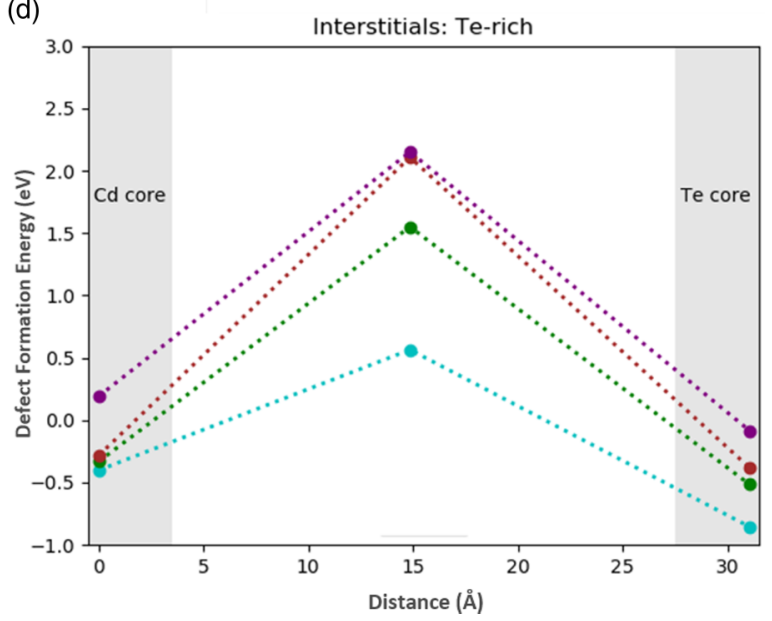

FIG. 12. DFE of $\mathrm{F}, \mathrm{Cl}, \mathrm{Br}$, and I as (a, b) Te substitutions and (c, d) interstitials, in the $\Sigma 3$ (112) GB structure in Cd-rich and Te-rich conditions. 
TABLE I. GB segregation energies of the halogens.

\begin{tabular}{lcc}
\hline \hline Element & $\begin{array}{l}\text { Segregation energy }(\mathrm{eV}) \\
\text { Te substitution }\end{array}$ & Interstitial \\
\hline $\mathrm{F}$ & -1.570 & -1.419 \\
$\mathrm{Cl}$ & -1.150 & -2.065 \\
$\mathrm{Br}$ & -1.001 & -2.489 \\
$\mathrm{I}$ & -0.798 & -2.234 \\
\hline \hline
\end{tabular}

the bulklike site there is a clear DFE trend of $\mathrm{F}>\mathrm{Cl}>\mathrm{Br}>$ I. Interstitials show the opposite trend.

These trends can be explained by considering the ionic radii of these elements. The ionic radii increase in the order $\mathrm{F}<\mathrm{Cl}<\mathrm{Br}<\mathrm{I}$. Iodine has a very similar ionic radius to tellurium. This suggests an $\mathrm{I}_{\mathrm{Te}}$ defect will occupy a very similar volume, distorting the structure less and therefore being most stable. Fluorine's ionic radius is the smallest at $59 \%$ of that of Te and so would be expected significantly to distort the structure as $\mathrm{F}_{\mathrm{Te}}$ and therefore be the least stable. However, a small ionic radius is beneficial as an interstitial because this reduces the surrounding distortion. Fluorine is therefore the most stable interstitial and iodine is the least stable interstitial.

The segregation energies to the most stable GB sites for all of the halogens are shown in Table I. The substitution and interstitial segregation energies decrease and increase, respectively, as halogen size increases. This again agrees with the ionic radius hypothesis.

In terms of experimental observations, all halogens would be expected to show strong segregation to GBs. Chlorine and bromine are especially similar as substitutions, which agrees with the work of Greenhalgh et al. [21]. It is possible that the larger halogens will penetrate further into grains as substitutions due to their lower segregation energies but the difference is unlikely to be observed due to the sensitivity of EDX elemental maps.
The electronic properties of other halogens have also been calculated for the TT2 position. This is the origin of the VBM+0.358- and +0.517-eV levels in the band gap. In Sec. III B it is shown that adding chlorine to this site reduces the DOS peaks within the band gap associated with this site but does not fully passivate the defect. As shown in Fig. 13, all halogens partially passivate this defect in the same way as chlorine. This suggests that all halogens are acting electronically similarly and would potentially be able to passivate GB defects.

It was shown in Sec. III B that both substitutions and interstitials are required for full passivation of the GB. While chlorine has an excellent balance of low DFE as Te substitution and interstitial defects it is possible to improve the stability of these defects by changing elements. A combined treatment of iodine, to form more stable Te substitutions and fluorine, to form stable interstitials, may improve the activation treatment by allowing the dopants to form the desired defects more easily. This may manifest as a lower treatment temperature, reduced cell cost, or greater concentration of dopants at GBs which would improve defect passivation and therefore efficiency. The GB structure used here is a simplified model. Real GBs are much more complex and thus have a greater concentration of defects in need of passivation, so an increased dopant concentration could be highly beneficial to cell performance.

\section{CONCLUSION}

In summary, chlorine has been shown to segregate strongly to $\Sigma 3$ (112) GBs as both $\mathrm{Cl}_{i}$ and $\mathrm{Cl}_{\mathrm{Te}}$. The increased segregation energy of $\mathrm{Cl}_{i}$ means its DFE is comparable with $\mathrm{Cl}_{\mathrm{Te}}$ in GBs in Cd-rich conditions and lower in Te-rich conditions. This means it is likely that both defects will exist at GBs rather than just the $\mathrm{Cl}_{\mathrm{Te}}$ considered by previous studies $[14,16,20,23]$.

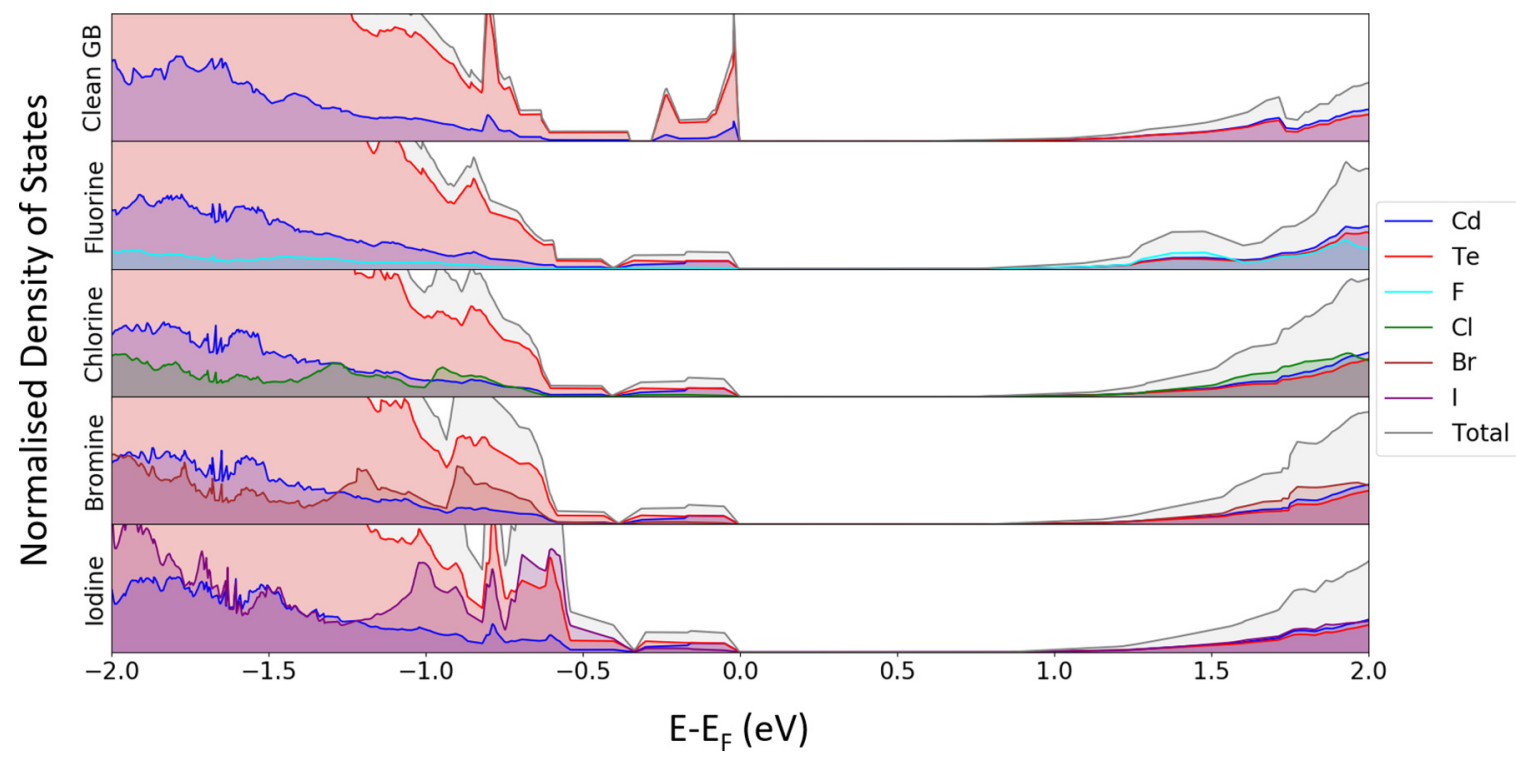

FIG. 13. Comparing DOS of $\mathrm{F}, \mathrm{Cl}, \mathrm{Br}$, and I at both TT2 sites, which is the origin of the $\mathrm{VBM}+0.358 \mathrm{eV}$ and $+0.517 \mathrm{eV}$ levels in the band gap, with the clean $\Sigma 3$ (112) GB. All halogens partially passivate this defect in the same way as chlorine. 
Four single-particle levels were identified in the GB's band gap which would significantly reduce cell efficiency through increased recombination. The VBM+0.358- and $+0.517-\mathrm{eV}$ levels are attributed to undercoordinated Te atoms at the TT2 site in the Te core while the VBM+0.239-eV energy level is bending up from the valence band and is attributed to the CT3 site Te-Te interaction in the Cd core. Chlorine substituting on the CT3 site fully passivates the VBM+0.239-eV level but substituting the TT2 site only partially passivates the VBM+0.358- and +0.517-eV levels. An additional chlorine interstitial in the Te core is required to fully remove the remaining levels.

The other halogens are shown to behave similarly to chlorine in terms of segregation to GBs and electronic effects in the GB. All are capable of partially passivating the VBM+0.358- and $+0.517-\mathrm{eV}$ levels by substituting the TT2 site. The DFE trends agree with the trend in ionic radius: $F$ is most stable as an interstitial while $\mathrm{I}$ is the most stable $\mathrm{Te}$ substitution.

To conclude, our DFT work advocates stable chlorine interstitials in the GB which lead to passivation of single-particle levels in the GB's band gap, thus improving cell efficiency on $\mathrm{CdCl}_{2}$ treatment.

\section{ACKNOWLEDGMENTS}

The authors acknowledge the use of Athena at High Performance Computing (HPC) Midlands+, which was funded by the Engineering and Physical Sciences Research Council (EPSRC) Grant No. EP/P020232/1, in this research, as part of the HPC Midlands+ consortium. The authors also recognize the use of the Hydra and LoveLace High Performance Systems at Loughborough University. Via our membership of the UK's High End Computing (HEC) Materials Chemistry Consortium, which is funded by EPSRC Grants No. EP/L000202 and No. EP/R029431, this work used the ARCHER UK National Supercomputing Service [27] and the UK Materials and Molecular Modelling Hub for computational resources, Materials and Molecular Modelling (MMM) Hub, which is partially funded by EPSRC Grant No. EP/P020194. The authors are also grateful to Prof. Kurt Barth and Prof. Walajabad Sampath of Colorado State University for long-term collaboration in thin-film CdTe solar cell research. They also wish to thank Dr. Buddhika Mendis of Durham University for the cathodoluminescence images and Dr. Kexue $\mathrm{Li}$ and Prof. Chris Grovenor of Oxford University for the nanoSIMS images.
[1] Photovoltaic Solar Energy Development, First solar CdTe photovoltaic technology: Environmental, health and safety assessment, 2013 (unpublished), https://www. firstsolar.com/en-Emea/-/media/First-Solar/SustainabilityDocuments/Sustainability-Peer-Reviews/Chile-PeerReview-Cener_EN.ashx.

[2] N. R. Paudel and Y. Yan, Appl. Phys. Lett. 105, 183510 (2014).

[3] M. Lingg, S. Buecheler, and A. N. Tiwari, Coatings 9, 520 (2019).

[4] T. A. M. Fiducia, B. G. Mendis, K. Li, C. R. M. Grovenor, A. H. Munshi, K. Barth, W. S. Sampath, L. D. Wright, A. Abbas, J. W. Bowers, and J. M. Walls, Nat. Energy 4, 504 (2019).

[5] J. Burst, J. Duenow, D. Albin, E. Colegrove, M. Reese, J. Aguiar, C.-S. Jiang, M. Patel, M. Al-Jassim, D. Kuciauskas, S. Swain, T. Ablekim, K. Lynn, and W. Metzger, Nat. Energy 1, 16015 (2016).

[6] I. Dharmadasa, Coatings 4, 282 (2014).

[7] J. Moseley, W. K. Metzger, H. R. Moutinho, N. Paudel, H. L. Guthrey, Y. Yan, R. K. Ahrenkiel, and M. M. Al-Jassim, J. Appl. Phys. 118, 025702 (2015).

[8] J. Moseley, P. Rale, S. Collin, E. Colegrove, H. Guthrey, D. Kuciauskas, H. Moutinho, M. Al-Jassim, and W. K. Metzger, J. Appl. Phys. 124, 113104 (2018).

[9] J. D. Major, Semicond. Sci. Technol. 31, 093001 (2016).

[10] A. Abbas, G. D. West, J. W. Bowers, P. Isherwood, P. M. Kaminski, B. Maniscalco, P. Rowley, J. M. Walls, K. Barricklow, W. S. Sampath, and K. L. Barth, IEEE Journal of Photovoltaics 3, 1361 (2013).

[11] D. Mao, C. E. Wickersham, and M. Gloeckler, IEEE Journal of Photovoltaics 4, 1655 (2014).

[12] T. A. M. Fiducia, K. Li, A. H. Munshi, K. Barth, W. S. Sampath, C. Grovenor, and J. M. Walls, in 2018 IEEE 7th World
Conference on Photovoltaic Energy Conversion (WCPEC) (A Joint Conference of 45th IEEE PVSC, 28th PVSEC \& 34th EU PVSEC), Waikoloa, HI (IEEE, Piscataway, NJ, 2018), pp. $1702-1706$.

[13] A. Abbas, B. Maniscalco, J. W. Bowers, P. M. Kaminski, G. D. West, and J. M. Walls, 2013 IEEE 39th Photovoltaic Specialists Conference (PVSC), Tampa, FL (IEEE, Piscataway, NJ, 2013), pp. 1930-1934.

[14] J.-H. Yang, W.-J. Yin, J.-S. Park, W. Metzger, and S.-H. Wei, J. Appl. Phys. 119, 045104 (2016).

[15] C. Li, Y. Wu, J. Poplawsky, T. J. Pennycook, N. Paudel, W. Yin, S. J. Haigh, M. P. Oxley, A. R. Lupini, M. Al-Jassim, S. J. Pennycook, and Y. Yan, Phys. Rev. Lett. 112, 156103 (2014).

[16] C. Y. Liu, Y. Y. Zhang, Y. S. Hou, S. Y. Chen, H. J. Xiang, and X. G. Gong, Phys. Rev. B 93, 205426 (2016).

[17] J. P. Perdew, Int. J. Quantum Chem. 28, 497 (1985).

[18] A. Shepidchenko, B. Sanyal, M. Klintenberg, and S. Mirbt, Sci. Rep. 5, 14509 (2015).

[19] M. J. Watts, T. A. M. Fiducia, B. Sanyal, R. Smith, J. M. Walls, and P. Goddard, J. Phys.: Condens. Matter 32, 125702 (2019).

[20] L. Zhang, J. L. F. Da Silva, J. Li, Y. Yan, T. A. Gessert, and S. H. Wei, Phys. Rev. Lett. 101, 155501 (2008).

[21] R. C. Greenhalgh, A. Abbas, A. H. Munshi, T. M. Shimpi, K. L. Barth, W. S. Sampath, J. W. Bowers, and J. M. Walls, in 2018 IEEE 7th World Conference on Photovoltaic Energy Conversion (WCPEC) (A Joint Conference of 45th IEEE PVSC, 28th PVSEC \& 34th EU PVSEC), Waikoloa Village, HI (IEEE, Piscataway, NJ, 2018), pp. 2990-2993.

[22] M. J. Watts, S. R. Yeandel, R. Smith, J. M. Walls, and P. M. Panchmatia, in 2018 IEEE 7th World Conference on Photovoltaic Energy Conversion (WCPEC) (A Joint Conference of 
45th IEEE PVSC, 28th PVSEC \& 34th EU PVSEC), Waikoloa, $H I$ (IEEE, Piscataway, NJ, 2018), p. 3884.

[23] C.-J. Tong and K. P. McKenna, J. Phys. Chem. C 123, 23882 (2019).

[24] J.-H. Yang, L. Shi, L.-W. Wang, and S.-H. Wei, Sci. Rep. 6, 21712 (2016).
[25] B. E. McCandless and J. R. Sites, Handbook of Photovoltaic Science and Engineering (Wiley, New York, 2003), pp. 617662.

[26] W. Shockley and W. T. Read, Phys. Rev. 87, 835 (1952).

[27] http://www.archer.ac.uk. 